\title{
THE INFLUENCE OF A HEAT TRANSFER COEFFICIENT PROBE ON FLUID FLOW NEAR WALL
}

\author{
Peter KOHÚT`, Otakar HOREJŠ, Martin MAREŠ
}

\begin{abstract}
Good knowledge of the convective boundary condition is necessary for finite element analysis of thermal deformation behavior in machine tools. There are a number of correlation equations for natural and forced convection and several correlations for mixed convection.
\end{abstract}

Due to a relatively wide range of dimensions, temperatures and speeds, all regimes of convective heat transfer can be observed in machine tools, including the transition region between laminar and turbulent free convection, characterized by Rayleigh number values ranging between $R a=10^{8}-10^{9}$. Since convection in machine tools is highly influenced by external and internal factors, the heat transfer coefficient characterizing convective heat transfer and its changes has to be evaluated experimentally.

An experimental technique for evaluating the heat transfer coefficient on the wall and its changes between the wall and the ambient air, based on an active sensor, is being developed. Since the probe dimensions are not negligible, given the fluid motion structures near the wall which are induced by buoyancy or by forced flow, the influence of the probe has to be considered. Paper deals with latest experimental results and summarizes previous work.

\section{INTRODUCTION}

As has already been mentioned in the abstract, convective heat transfer is now necessary for modeling the thermal behavior of machine tool structures - unlike about two decades ago, when convective heat transfer was mostly neglected as a source of machining error. Thermal effects on machine tools cause up to $70 \%$ of machining inaccuracy [1]. The modeling of thermal deformation is a very important instrument for increasing the accuracy of the machining process. Thermal simulation tools are used to reduce the number of experiments and prototypes, as well as costs. The main problem of the simulation approach is the requirement to implement well-established boundary conditions.

Interaction between the machine tool structure and the ambient air is usually modeled with convective and radiation heat transfer mechanisms. The complexity of convective heat transfer lies in the value of the heat transfer coefficient (HTC), and a number of correlation equations are available in the literature, e.g. [2].

\footnotetext{
- Peter Kohút, Horská 3, 12800 Praha 2, P.Kohut@rcmt.cvut.cz Otakar Horejš, Horská 3, 12800 Praha 2, O.Horejs@rcmt.cvut.cz Martin Mareš, Horská 3, 12800 Praha 2, M.Mares@rcmt.cvut.cz
}

This is an Open Access article distributed under the terms of the Creative Commons Attribution License 2.0, which permits unrestricted use, distribution, and reproduction in any medium, provided the original work is properly cited. 
Due to the complexity of machine tool geometry and the low predictability of conditions during the machining process, the correlations can only be adopted to a limited extent. It is therefore necessary to develop techniques for experimental evaluation of HTC.

In our earlier works [3], we described a measurement procedure for HTC identification in open moving air, using a probe in the form of a heated flat plate located in a channel. This was followed by an HTC probe developed to take into account the conditions of measuring the wall heat transfer coefficient in machine tools [4].

The method for measuring the wall HTC and its changes in machine tools is based on the presumption that the probe has a local influence on the fluid motion near the wall and, consequently, on the wall heat transfer. This influence has to be estimated under the conditions of forced as well as natural convection.

The influence of the sensor itself on the fluid flow near the wall is discussed in the following sections.

\section{EXPERIMENTAL SETUP}

A set of visualization experiments was performed in a closed-circuit wind tunnel with an open test section, in order to identify the character of the local fluid flow near the HTC probe. This was followed by a set of visualization experiments on a vertical heated plate, under free convection conditions.

The glycerin smoke visualization technique was adopted in the forced flow case, where the heated wire, together with the oncoming velocity vector, defined the vertical symmetry plane of the wind tunnel test section. A horizontal plate with the dimensions of $300 \mathrm{~mm}$ span-wise and $400 \mathrm{~mm}$ stream-wise, representing the wall, was located in the center of the wind tunnel test section, and a HTC probe was fixed on or near the vertical plane of symmetry of the test section, ca. $130 \mathrm{~mm}$ from the aerodynamically shaped leading edge of the plate which had rounded corners. The HTC probe was in the shape of a cylinder $8 \mathrm{~mm}$ high, with $40 \mathrm{~mm}$ in diameter and a rounded upper edge with a fillet (Figure 1).

The flow near the probe was studied for oncoming velocity range of approximately $V=(1.7-2.5) \mathrm{m} / \mathrm{s}$ which corresponds to stream-wise length based Reynolds number range $R e_{\mathrm{L}}=(45000-67000)$.

A vertical plate with the dimensions of $970 \times 560 \mathrm{~mm}$ (vertical $\times$ horizontal) was equipped with a defined heat source (heater) in the free flow case. The HTC probe was located in the center of the vertical plate. Visualization smoke was generated using a wooden stick located $270 \mathrm{~mm}$ downwards from the HTC probe, and between 0 and 10 $\mathrm{mm}$ from the heated plate (Figure 1). The wall temperature and the ambient temperature were measured under three different heating regimes. These regimes were chosen in such a way as to cover the range of the Rayleigh number $\mathrm{Ra}=\left(9.2 \cdot 10^{7}-\right.$ $\left.1.7 \cdot 10^{9}\right)$, and the vertical dimension of the plate was considered as the characteristic length.

The measured quantities included oncoming velocity, heater input power, and the temperatures of the probe, the heated wall, and quiescent air. The uncertainty of the temperature and velocity measurements was up to $0.5{ }^{\circ} \mathrm{C}$ and $0.2 \mathrm{~m} / \mathrm{s}$ respectively, using a K-type thermocouple measurement device and a rotating vane anemometer (Ahlborn). The visualization was recorded with a standard camera (Canon EOS 450D) in two regimes - a short exposure time with flash, and a long exposure time without flash. 
Schematic views of both experimental setups are depicted in Figure $\mathbf{1}$ as well as the HTC probe itself, where $\mathrm{V}$ stands for the oncoming velocity vector and $\mathrm{G}$ for the vector of gravity.
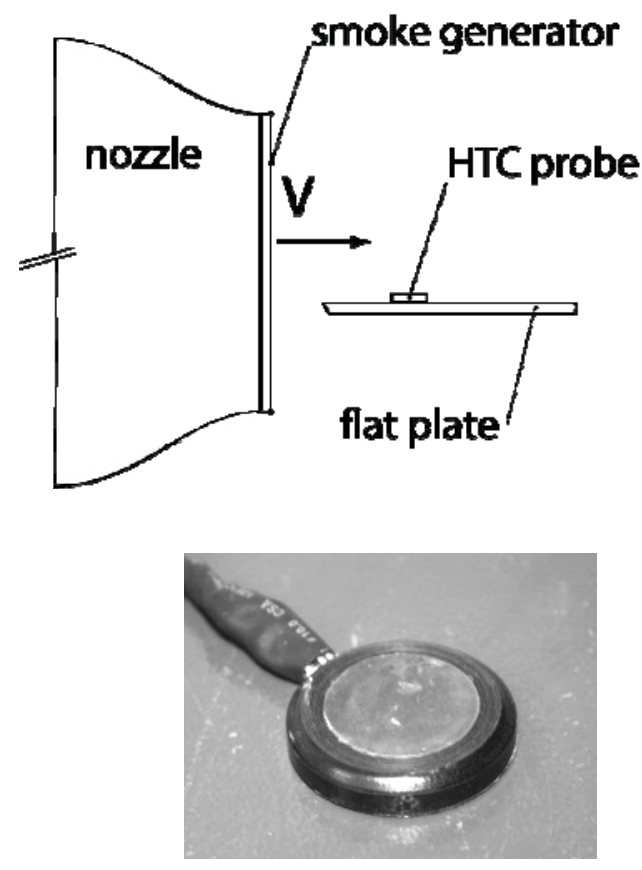

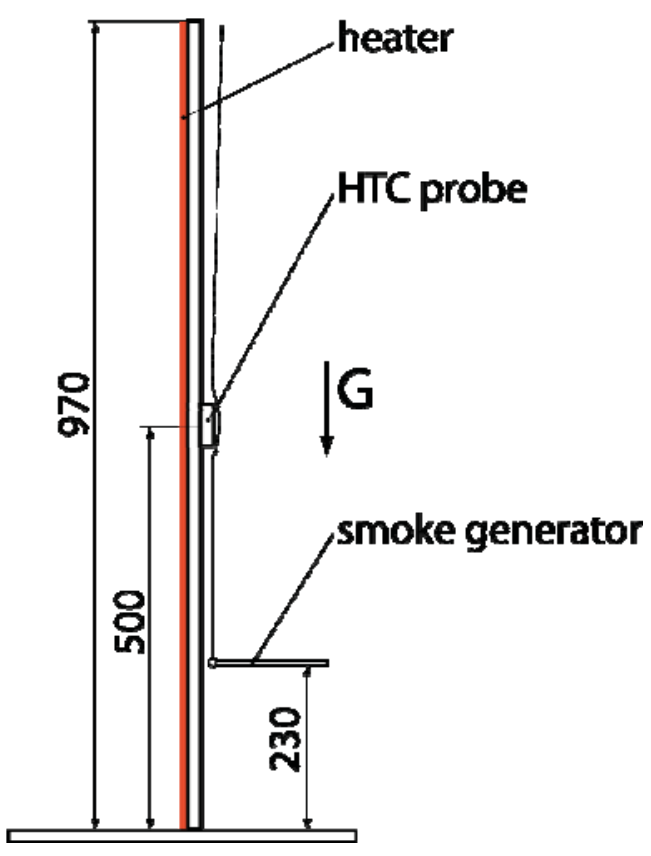

Figure 1: Experimental setup - horizontal forced flow and HTC probe (left) and natural convection on vertical plate (right)

\section{ResULtS AND Discussion}

\section{Forced flow}

Several examples of flow visualizations performed under forced flow conditions are shown in Figure 2 and Figure 3.

According to [5], at $R e_{\mathrm{d}}=(40-150)$, called the stable range, regular vortex streets are formed and no turbulent motion develops. In the stable range the vortices decay by viscous diffusion.

Glycerin smoke is vaporized from the heated wire in evenly spaced locations on this wire due to two-wire spiral construction. The Reynolds number based on this diameter reaches the values of $R e_{d}=(50-150)$ within the range of the oncoming velocities used and with the wire diameter of approximately $d=0.5 \mathrm{~mm}$. These values are within the range in which the von Kármán vortex path can be observed. This phenomenon was probably observed when short exposure times and flash were used for the recording. The observation of such structures and their behavior in the vicinity of the wall and the HTC probe was used to interpret the local character of the flow.

Long exposure times showed time-averaged particle tracks characterizing the flow field pattern and were used to describe the overall influence of the probe on the flow near the wall. Some figures are blurred in the direction from the center to the horizontal edges, which is generally due to the depth of field. 
The structure of the flow generated by the interaction between the oncoming flow and the heated wire in the form of a vortex path remained the same when the flow was passing the probe and even after it was influenced by the probe, as can be seen in the presented Figure 2 (b, d) and Figure $\mathbf{3}(a, b, c)$.

Figure $2(a, c)$ shows the long exposure records where a small recirculation region can be identified near the rear end of the probe as well as near the front part of the probe. Reattachment of the flow to the plate occurs at a distance of less than one probe diameter from the probe. The influence of the mounting device behind the probe is considered to be negligible with respect to the character of the flow near the probe and its influence on convective heat transfer.

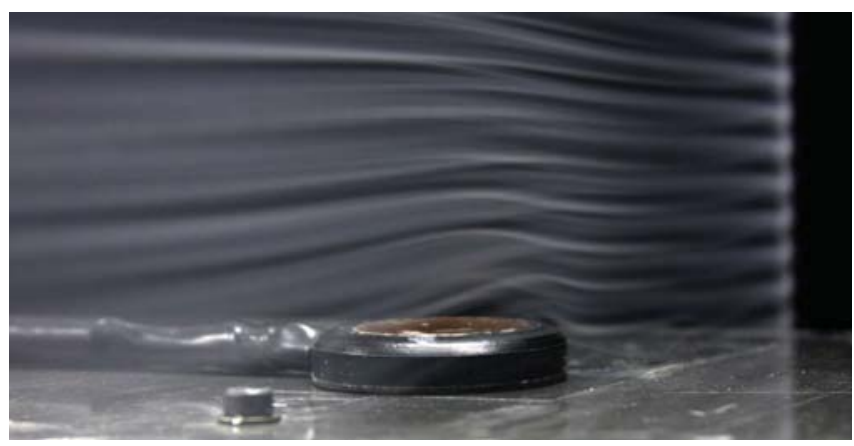

$a-\operatorname{Re}_{\mathrm{L}}=45000$, long exposure, no flash

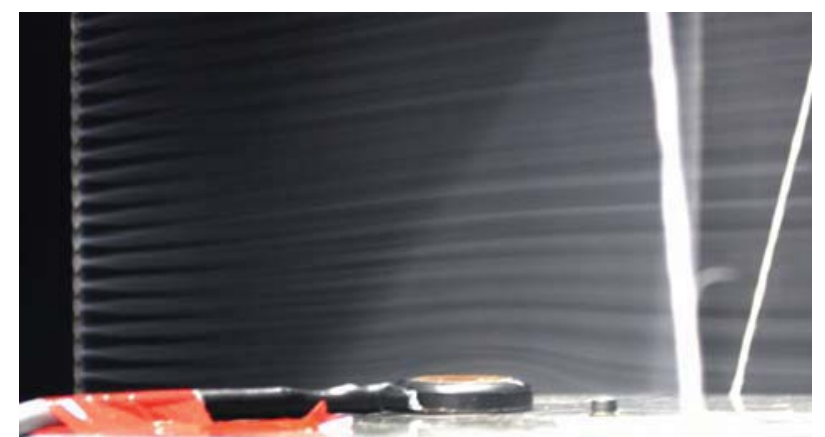

$c-\operatorname{Re}_{\mathrm{L}}=67000$, long exposure, no flash, wake region

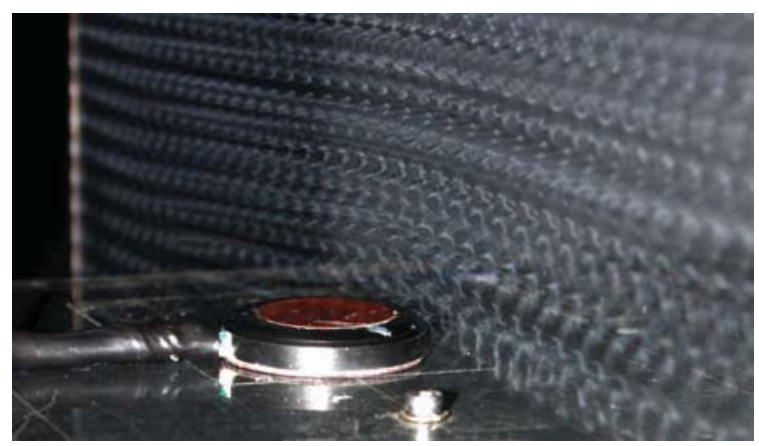

b $-R_{\mathrm{L}}=56000$, short exposure, flash Off-axis flow near the probe

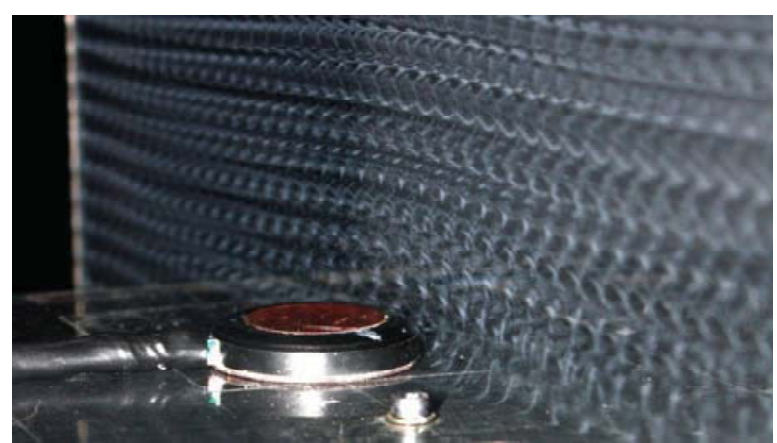

d $-\mathbf{R e}_{\mathrm{L}}=56000$, short exposure, flash Off-axis flow near the probe

Figure 2: Flow patterns for various $\mathbf{R e}_{\mathrm{L}}$

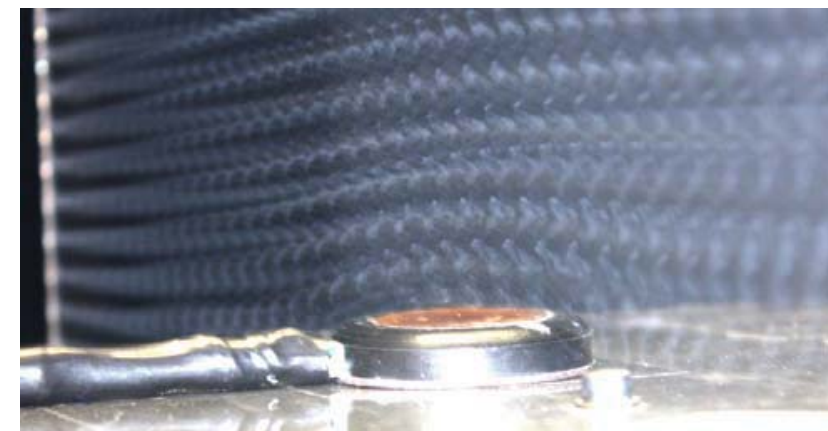

$a-\operatorname{Re}_{\mathrm{L}}=67000$, short exposure, flash

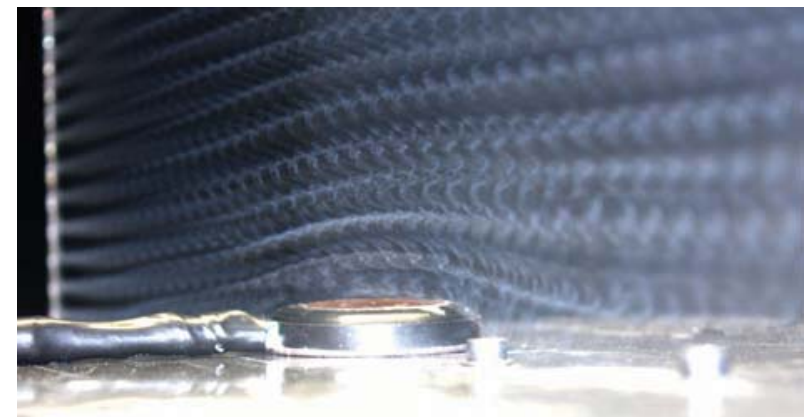

b $-\operatorname{Re}_{L}=67000$, short exposure, flash 


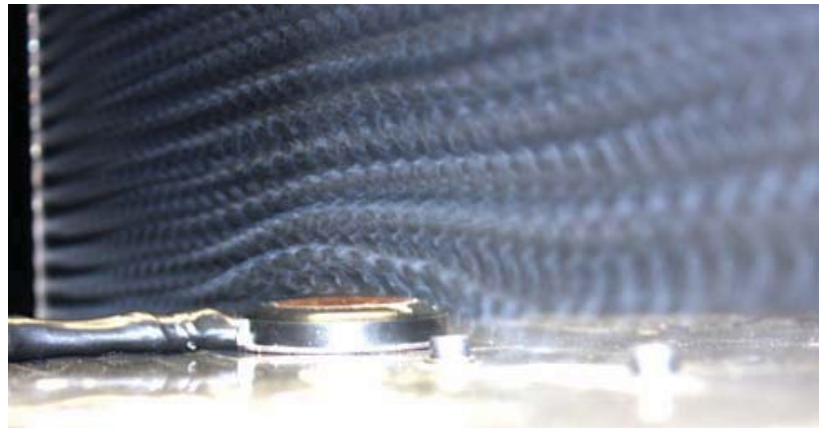

$c-\operatorname{Re}_{\mathrm{L}}=67000$, short exposure, flash

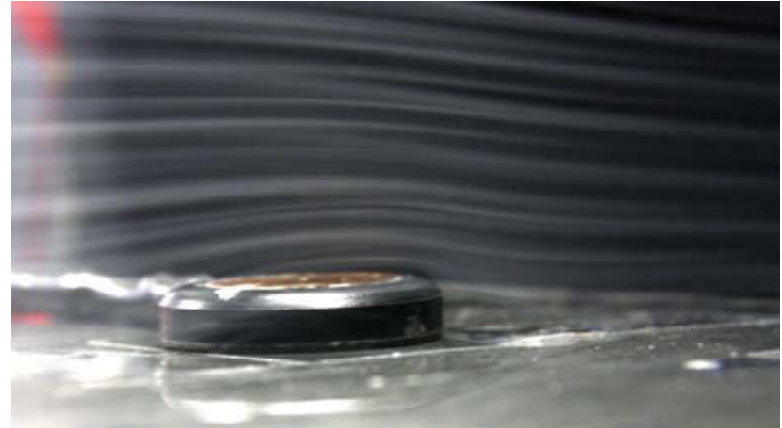

d $-\operatorname{Re}_{\mathrm{L}}=67000$, long exposure, no flash

Figure 3: Flow patterns for $\mathrm{Re}_{\mathrm{L}}=67000$, without flash, long exposure time.

\section{Free convection}

Figure 4 depicts the flow pattern near the probe under free convection conditions. No change in the flow pattern character was observed in the vicinity of the probe. It can therefore be said that the probe probably had almost no influence on the free convection flow pattern. This is valid for the whole range of the Rayleigh numbers tested. It has to be said, however, that the stream of smoke particles used for visualization was very sensitive to external influences.

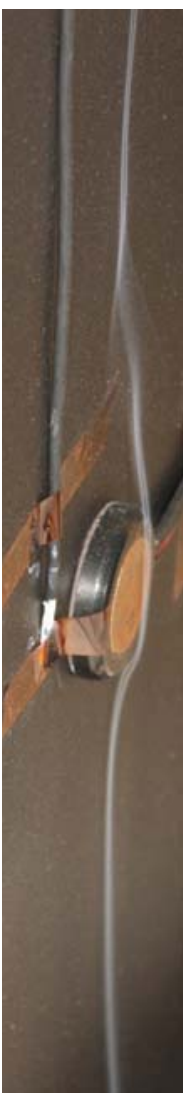

a

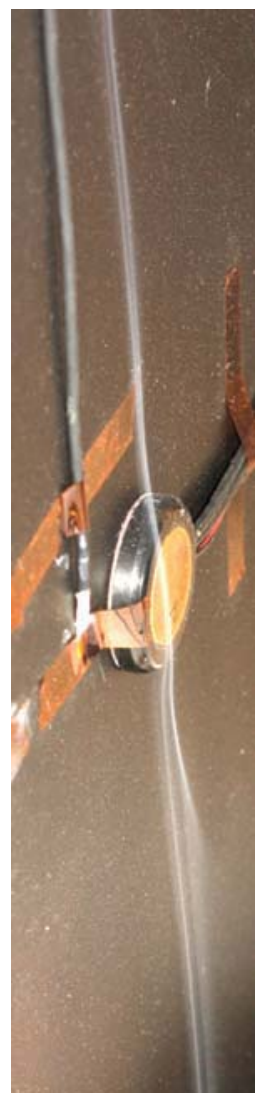

b

Figure 4: Free convection over heated vertical plate, $R a_{L}=1.8 \cdot 10^{9}$ 
The influence of the visualization technique itself on the induced flow has to be considered as it generates a considerable amount of heat. The total amount of heat and the influence on the flow pattern far from the source is considered as small, due to the small dimensions of the heat source. The flow pattern near the HTC probe is the most important for the case of free convection.

Figure 4 shows examples of visualization of flow over the vertical flat plate under free convection conditions.

\section{Conclusions}

Visualization experiments were performed to verify the presumption of local influence of the HTC probe on the fluid flow near the wall under forced as well as free convection conditions.

According to the experiments performed it can be stated that:

- The presence of the HTC probe had no strong influence on the flow pattern near the wall in the tested range of $R e_{L}=(45000-67000)$ under forced flow conditions, where the visualization technique used provides valuable records.

- Small separation regions were observed near the front and the rear part of the HTC probe, with dimension equaling approximately $1 / 2$ and 1 probe diameter respectively. However, these observations are influenced by the limits of the technique used; it can therefore be said that the separation or recirculation regions are not larger than the observed values which represent the most likely upper limit of the real value.

- The structure of the vortex path is not influenced strongly by the presence of the HTC probe on the plate, which confirms that there is little influence on the local character of the flow near the probe. The vortex structures are sustained in the flow, even when they pass the region influenced by the probe.

- The range of the velocities corresponds to the values usually observed in machine tool applications, except the case of regions that are close to cutters, spindles etc.

- The influence of the probe is negligible under free convection conditions within the tested range of the Rayleigh criterion, provided that the influence of the method itself can be neglected.

- The influence of the HTC probe on the fluid flow pattern near the wall can be considered as local or negligible for the tested range of the Reynolds and Rayleigh criteria.

\section{REFERENCES}

[1] Bryan J.: International Status of Thermal Error Research (1990), CIRP annalsmanufacturing technology, vol. 39/2, 1990, pp. 645-656

[2] Incropera F.P., DeWitt D.P.: Fundamentals of heat and mass transfer, $5^{\text {th }}$ ed., Wiley\&Sons, 2002

[3] Kohut P., Barta P., Horejs O.: Heat transfer coefficient evaluation procedure, In Proceedings of the $19^{\text {th }}$ International Symposium on Transport Phenomena, Reykjavík, Iceland, 2008

[4] Kohut P., Horejs O., Mares M.: Experimental Identification of Convective Heat Transfer in Machine Tools, In Proceedings of World Congress of Engineers, International Conference of Mechanical Engineering, vol. 3, IAENG, 2011, pp. 2122-2125

[5] Roshko A.: On the Development of Turbulent Wakes from Vortex Streets, NACA report 1191, 1954, pp. 1-25 